\title{
Nonlinear Analysis of Cardiac Optical Mapping Data Reveals Ordered Period in Defibrillation Failure
}

\author{
JD Simonotto $^{1}$, MD Furman ${ }^{1}$, WL Ditto ${ }^{1}$, ML Spano ${ }^{2}$, \\ $\mathrm{G} \mathrm{Liu}^{3}, \mathrm{KM}$ Kavanagh ${ }^{3}$ \\ ${ }^{1}$ Biomedical Engineering Dept, University of Florida, Gainesville, FL, USA \\ ${ }^{2}$ US Navy, Carderock Laboratory, W Bethesda, Maryland, USA \\ ${ }^{3}$ Department of Medicine, University of Alberta, Edmonton, Alberta, Canada
}

\begin{abstract}
A high-speed video camera and voltage-sensitive dyes were used to acquire high resolution (80x80 pixels) and high-speed (500 us/frame) optical signals of ventricular fibrillation in a Langendorff-perfused porcine heart. The resulting spatiotemporal dynamics were recorded before and after the application of a defibrillation shock in order to study the mechanism of defibrillation failure. We used recurrence plots ass a tool to qualify the evolution of ordered behavior on the heart surface before fibrillation was reestablished in defibrillation failure. Such ordered periods may point to robust periods in which the defibrillation attempt has had the most effect and may provide a window in which a smaller, corrective shock may be applied to achieve defibrillation.
\end{abstract}

\section{Introduction}

Ventricular fibrillation (VF) is a serious medical problem; in the western world, VF claims more lives than any other heart disease. But little is understood about how normal, ordered behavior (sinus rhythm) can suddenly change into the complex activity that characterizes VF. It has been established, that when there are wavebreaks on the surface of the heart (a literal breakup of the smoothly propagating electrical wavefront), spiral waves can result. [1,2] Further breakdown can lead to VF, but it is unclear exactly how changes in the system can lead to such a breakdown. Currently the only effective therapy for VF is the delivery of a rather large electrical shock (stimulus) across the myocardium with the goal of terminating $\mathrm{VF}$ and allowing the sinus node to reestablish sinus rhythm. However, external defibrillation shocks (DS), which for humans can be 200 - 360 Joules in magnitude, are not always successful. It is important to understand why defibrillation failure occurs in order to make DS more effective. Experimentally recorded data from porcine ventricles (taken from a whole heart preparation, but with the main focus on the ventricles) undergoing repeated defibrillation attempts was analyzed to understand the mechanism of defibrillation failure.

\section{Methods}

High spatial and temporal resolution optical mapping techniques and voltage sensitive dyes were used to visualize the surface excitations of porcine heart. The data were taken at 1000 frames per second by a Red Shirt Imaging CCD camera with an $80 \times 80$ pixel resolution covering a $5 \times 5 \mathrm{~cm}$ area.

All animal procedures received approval from the Health Sciences Animal Welfare Committee of the University of Alberta. The heart was rapidly removed and submerged in cool perfusion fluid. The right and left coronary arteries were cannulated with DLP arteriotomy canulae. Coronary flow was adjusted between 90 and $120 \mathrm{ml}$ per minute per $100 \mathrm{~g}$ to maintain a mean perfusion pressure of $70-80 \mathrm{~mm} \mathrm{Hg}$. A Millar catheter was used to monitor the perfusion pressure. Perfusate temperature was regulated at $37.5 \mathrm{C}+/-0.5 \mathrm{C}$ by water jacketed heat exchangers. Temperature was monitored via thermisters located in the water jacket perfusion line and the perfusate inlet port. An intramural temperature probe was inserted near the left ventricular apex. [3] The perfusion connector, on to which the coronary artery canulae were subsequently attached, contained additional temperature control injection ports for infusion of electromechanical dissociation agents and voltage sensitive dye. This proximity of the dye injection site and the coronary artery arterial tree minimizes the dead space for possible dye binding to red blood cell membranes and to blood proteins. The heart was stained with a $100 \mu \mathrm{M}$ solution of di-4-ANEPPS was infused at $1 / 10$ of the tissue flow rate for an infusion rate of $10 \mu \mathrm{M} / \mathrm{min}$. The electromechanical dissociation agent Cytochalasin D was infused at $1 / 10$ the arterial flow rate to eliminate movement artifact.

An extracellular mapping system was synchronized with the optical mapping system to continuously monitor atrial and ventricular rhythms during optical mapping. It was also possible to deliver electrical current through these electrodes to facilitate atrial and ventricular pacing. The extracellular electrodes were positioned on the posterior surface of the heart to avoid obstructing optical 
mapping. Titanium mesh defibrillation patch electrodes were positioned on the right atrium (surface area of 2.3 $\mathrm{cm}^{2}$ ) and the apex of the left ventricle (surface area 5.8 $\mathrm{cm}^{2}$ ) to facilitate defibrillation.

If the heart was not initially in ventricular fibrillation it was induced with rapid ventricular pacing via the extracellular electrodes. An external defibrillator (Ventritex HVS-02, Ventritex Inc.) was used for defibrillation. A single capacitor biphasic waveform was used in which the leading edge voltage of phase 2 was equal to half the leading edge voltage of phase one. Each phase of the waveform was $6 \mathrm{~ms}$ in duration. Defibrillation energies were delivered through external patches, which were placed on the area of the right atrium and LV apex. The initial shock delivered was a low energy shock with first and second phase amplitudes of 200 and 100 volts respectively. If the initial shock was unsuccessful, the strength of the next and subsequent shocks was increased by $50 \mathrm{~V}$ until defibrillation was achieved. [4] Defibrillation threshold was defined as the lowest voltage and current that achieved defibrillation.[4]

Due to memory restrictions, the data was limited to 10 seconds of recording at $1000 \mathrm{fps}$, sufficient to cover the transition from fibrillation $\rightarrow \mathrm{DS} \rightarrow$ defibrillation failure. The data was processed with a custom image processing program as described below.

After the camera system acquires the data, it is stored in a proprietary format. Subsequently we read in the raw data in preparation for further processing. At this stage we may average either 2 or 4 frames of the raw data, giving effective frame rates of $500 \mathrm{fps}$ or $250 \mathrm{fps}$. From this data we stretch the dynamic range of each pixel to remove effects due to uneven illumination and non-planar geometry, subtract a global baseline value, and then "debleach" the data by detrending the pixel values and expanding the dynamic range on a frame by frame basis. A typical number of frames used is 9216. Time series were extracted from individual pixels of the processed data for analysis.

Movies were made of the processed data, showing electrical excitations over the heart surface. For one movie, four frames of the data were averaged together to give a more well-defined wavefront (wave of excitation in the tissue), but effectively reducing the frame rate to 250 fps. Space-time volume plots were made from the frameaveraged processed data (Fig.1). In these plots one can see several distinct stages in the evolution of the data. Initially the electrical activity was established VF, with multiple waves coexisting on the tissue surface in a complex manner (Fig. 1, upper left). The DS came approximately two seconds into the data recording, but, before the shock could travel across the entire tissue surface, a spontaneous reentry wave originating in the lower left portion of the tissue collided with the defibrillation wave. We refer to the portion of tissue from which the spontaneous wave originated as the reentry area. There was no reentry resulting from this collision, and regular excitation waves propagate across the surface from the bottom edge of the frame to the top edge in normal fashion, but there is a tissue path (from the bottom right to the upper middle) in which waves of excitation travel more quickly compared to the rest of the tissue. After several excitations (one sec), waves are generated from this "fast" area (Fig. 1, upper right); we refer to this

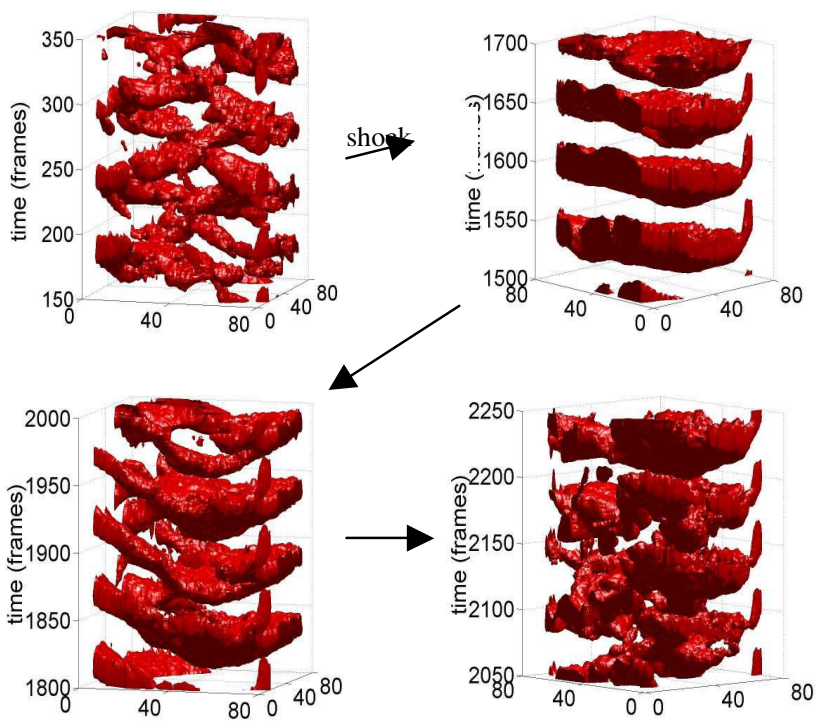

FIGURE 1. Space-time volume plots of processed data as described in the text. The $\mathrm{z}$-axis is time (frames, where 1 frame $=2 \mathrm{msec}$ ) and the $\mathrm{x}$ - and $\mathrm{y}$-axis are heart surface area (80x80 pixels covering a $5 \times 5 \mathrm{~cm}$ area). Note that the viewing angle varies in order to highlight features of interest. The upper left figure shows activations from frames 150 to $350(0.3$ to $0.7 \mathrm{sec})$ showing reentry activity in the form of spatially discontinuous waves. Note the temporal periodicity present in the waves spatially; i.e., some waves occur again and again in the same manner in the same place (meaning that multiple waves coexist in a stable manner with each other). The upper right figure shows ordered behavior from frames 1500 to 1700 (3.0 sec to $3.4 \mathrm{sec}$ ) which occurs after the defibrillation shock (DS) and is due to excitation wave generation (focal activity) within the frame of visible tissue. The lower left figure shows activations from frames 1800 to 2000 (3.6 sec to 4.0 $\mathrm{sec}$ ) and exhibits reentry in the form of bridges connecting one full-tissue activation to the next. The lower right figure shows activations from frames 2050 to 2250 (4.1 sec to 4.5 $\mathrm{sec}$ ) and emphasizes the breakdown of ordered activity within the full-tissue activations, with reentry connections in some areas of the tissue. Some of the tissue behavior is similar to the spatially discontinuous waves showing temporal periodicity that marked the "chronic" VF at the beginning of the dataset (upper left). 
area as the focal area (as it is a focus of activity generation). The movie then chronicles which type of activity is seen in the rest of the tissue. One can see regular activity induced by the focal area as well as the beginning of reentry activity, seen as "bridges" connecting one whole-surface activation to the next (Fig. 1 , lower left). As the movie ends, reentry is the more established type of activity on the heart surface (Fig. 1, lower right). One can see that defibrillation has failed because complex paths of reentry present at the beginning of the movie (Fig. 1, upper left), in which multiple wavelets coexist with each other in a stable manner, begin to reassert themselves at the end of the dataset (Fig. 1, lower right).

\section{Recurrence plots and results}

Kac first introduced recurrence plots in 1947 with respect to discrete stochastic processes. In the 1980's, Eckmann, et, al. [5] published a paper examining recurrence plots of dynamical systems and thereby introducing recurrence plots to the field of nonlinear dynamics. Recurrence Plots, as described in (1), display the dynamical phase relationships in signals in the $\mathrm{n}$ dimensional phase space [6]. They are relatively simple to generate using two time series, either identical or different. The series are assigned one to the $\mathrm{x}$-axis and one to the y-axis. The two-dimensional xy-plane is therefore a thresholded difference plot of time series over all points.

$$
R_{l m}^{i j}=\Theta\left(\varepsilon-\left\|s_{i j}\left(k_{l}\right)-s_{i j}\left(k_{m}\right)\right\|\right) \quad l, m=1, \ldots, L
$$

The Euclidian distance of the difference of two points is subtracted from a threshold.

The matrix of recurrence points $R_{l m}^{i j} \quad$ creates patterns that illustrate the association between the two time series. The Euclidian distance becomes the absolute value of the difference and the 2D-matrix formed by the differences can be any positive rational number - where zero indicates parity between the two waveforms. Lines parallel to the diagonal indicate similarities in the two waveforms with a temporal shift equal to the difference in the $\mathrm{x}$ and $\mathrm{y}$-coordinate at the point of interest - this is true even for disparate waveforms. Different patterns appearing in recurrence plots have different meanings: A random time series will exhibit a random distribution of points with few contiguous points, while a time series with repeating visits in phase space will generate lines parallel to the diagonal.

An example recurrence plot is generated with the same defibrillation failure time series signal projected along both the $\mathrm{x}$ and $\mathrm{y}$-axis in Fig 2 and illustrates a typical characteristic for non-deterministic signals (the time series is plotted on the upper portion of the figure). The diagonal is zero since the two signals are the same for all t. Interestingly, we see two areas on this figure which indicate a transition, one of which is the defibrillation shock, the other being a more subtle feature not notable in a movie of the data, indicating a change of ordering when the dominant features of the system go from focal beating to general reentry. It is this secondary changeover that may indicate an appropriate time to administer a smaller, better timed corrective shock to try to defibrillate again, rather than administer a full-strength shock again, causing possible tissue damage.

\section{Discussion and conclusions}

We have observed the evolution of failed defibrillation and spontaneous focal beating using cardiac optical mapping. One can clearly see periods in which the entire tissue behaved similarly to the focal area and the gradual breakdown of this type of behavior, with reentry gradually becoming the more dominant type of behavior towards the end of the dataset. This switchover is captured in Recurrence Plots, showing a change in order,
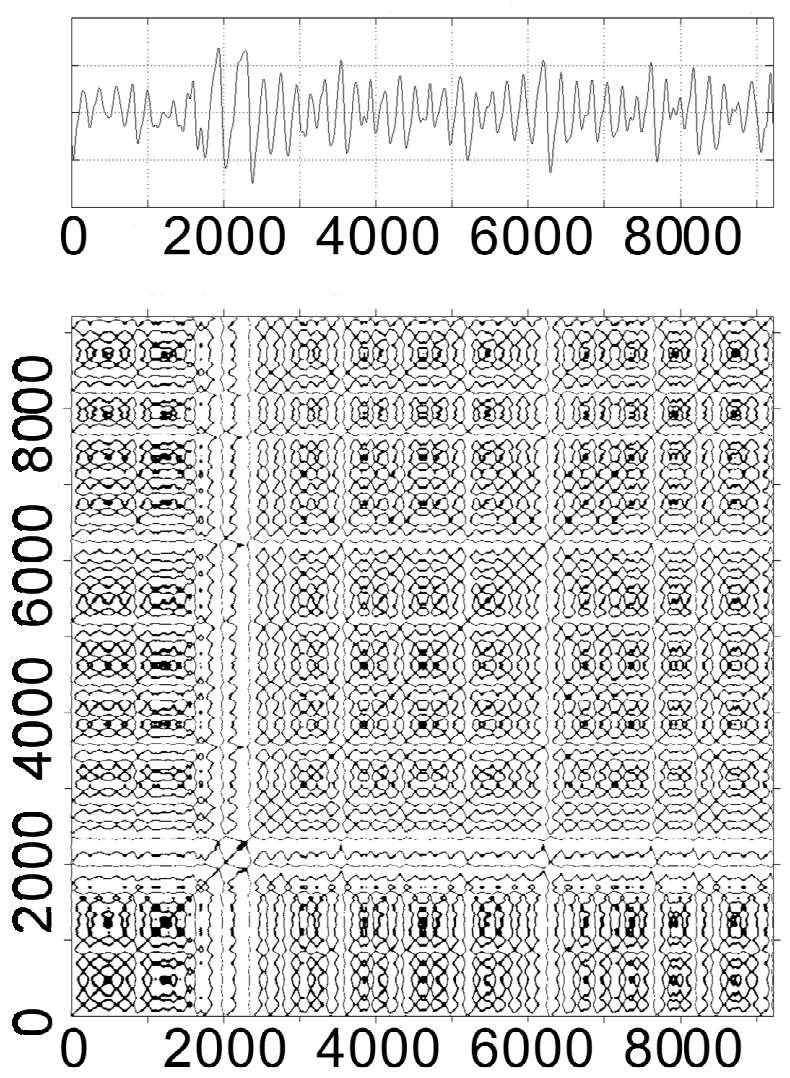

FIGURE 2 Recurrence plot of a time series taken from a defibrillation failure dataset, showing two areas of transitional organization. The first area is, understandably the defibrillation shock, but the second, surprisingly, occurs as there is a spontaneous global transition from focal-driven activity to reentry behavior. 
which can be interpreted as a time of maximal orderedness that can be determined with analysis of very short noisy datasets (a strength of such plots). It can be extrapolated that this time in which order was increased could be a window in which a minimal corrective shock could be applied to a failed cardioversion and restore the entire tissue to order. What we found was a robust marker to demonstrate when a corrective shock could be applied with the energy landscape was at its lowest point, in agreement with several points of defibrillation failure. We have yet to characterize how the changeover from focaldriven to reentry activity takes place exactly, but in future analysis we believe that using the spatial distribution of synchronization index changes over time to determine optimal windows for cardioversion should yield promising results.

The implications of this work are potentially profound, with minimally invasive 'smart' cardioversion being the end goal of many researchers all over the world. Current internal and external defibrillators simply deliver enough energy to rapidly excite and de-excite cardiac tissue blindly, allowing normal sinus rhythm to reinitialize a beat; the main difference is currently the amount of voltage required by each device. Typically the voltage settings are left up to the operator in the case of exernal defibrillatiors. With a consistent measure for determining relative order in the complex spatio-temporal dynamics, perhaps a minimal but properly timed cardioversion shock would be more effective. This would mean less damage to tissue due to excess voltage applied or multiple shocks applied, less pain, and equal if not greater success rate in cardioversion.

\section{Acknowledgements}

We would like to thank Sheri Prucka for the monetary and scientific support. We would also like to thank Dr. Michael Shlesinger and the US Office of Naval Research for support.

\section{References}

[1] Winfree, A.T. Science 266, 1003-1006 (1994).

[2] Witkowski, F.X., Leon, L.J., Penkoske, P.A., Giles, W.R., Spano, M.L., Ditto, W.L., and Winfree, A.T., Nature 392, 78 (1998).

[1] Witkowski, F.X., Plonsey, R., Penkoske, P.A. \& Kavanagh, K.M. Circ Res 74, 507-524 (1994).

[2] Feeser, S.A. et al. Circ. 82, 2128-2141 (1990).

[3] Zbilut, J.P. and C.L. Webber, Phys. Lett A, 1992. 171: p. 199.

[4] Webber, C.L., et al., Elucidating protein secondary structures using alpha-carbon recurrence quantifications. Proteins-Structure Function and Genetics, 2001. 44(3): p. 292303.
Jennifer Simonotto

Biomedical Engineering Department

University of Florida

130 BME Building, PO Box 116131

Gainesville, Florida, 32608

USA

jennifer@bme.ufl.edu 